\title{
Manejo perioperatorio del paciente con coagulopatía por COVID-19
}

\section{Perioperative management of the patient with COVID-19 coagulopathy}

Dra. Salomé Alejandra Oriol-López*

RESUMEN. La aparición de la enfermedad por coronavirus SARS-CoV-2, en China a finales de 2019, se ha diseminado rápidamente hasta convertirse en pandemia, cuyas características clínicas varían desde ser asintomático hasta tener la forma grave, donde los pacientes cursan con alteraciones de la coagulación, generalmente un estado hipercoagulable, pueden evolucionar a coagulación intravascular diseminada, falla orgánica múltiple y/o fallecimiento. Es importante investigar comorbilidades; verificar el estado de coagulación desde el primer contacto, analizando las vías: endógena, exógena, común, sistema fibrinolítico y recuento plaquetario, haciendo uso de la tecnología como la tromboelastografía y el sonoclot. El uso de anticoagulantes está indicado sobre todo la heparina no fraccionada o la de bajo peso molecular, ajustando dosis con exámenes de laboratorio, para evitar estados de hipocoagulabilidad, emplear hemoderivados cuando sea necesario. Recordar que el tratamiento antiviral puede tener interacciones con los anticoagulantes, sobre todo los orales. No es común el sangrado. Existen problemas de oclusión de catéteres instalados, evitar el lavado frecuente de los mismos con heparina. Si el paciente requiere de procedimiento quirúrgico o invasivo de emergencia, verificar la evolución de la coagulación, si no se puede postergar, emplear el tratamiento con hemoderivado de acuerdo a la alteración detectada. Seguir el algoritmo de manejo en el quirófano para evitar contagios del personal de la salud.

ABSTRACT. The appearance of the SARS-CoV-2 coronavirus disease, in China late 2019, has spread rapidly to become a pandemic, the clinical characteristics which range from being asymptomatic to having the severe form, where patients present abnormalities of the Coagulation, generally a hypercoagulable state, may evolve to disseminated intravascular coagulation, multiple organ failure, and / or death. It is important to investigate comorbidities; verify the coagulation status from the first contact, analyzing the routes: endogenous, exogenous, common, fibrinolytic system and platelet count, using technology like thromboelastography and sonoclot. The use of anticoagulants is indicated, it can be used unfractionated or low molecular weight heparin, with laboratory tests to adjust the dose, in order to avoid states of hypocoagulability, use blood products when necessary. Remember that antiviral treatment can have interactions with anticoagulants, especially oral ones, bleeding is not common. There are occlusion problems of installed catheters, one must avoid frequent of these washing with heparin. If the patient requires an emergency surgical or invasive procedure, it must verify the evolution of the coagulation, if it cannot be postponed, use treatment with blood products according to the alteration detected. Follow the management algorithm in the operating room to avoid infections from health personnel.
Anestesiología

Octubre-Diciembre 2020

Vol. 43. No. 4. pp 257-263

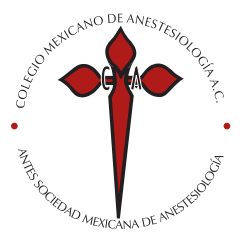

Palabras clave:

Coagulopatía, anticoagulantes.

Keywords:

Coagulopathy, anticoagulant.

* Médica Anestesióloga,

Hospital Juárez de México.

Solicitud de sobretiros: Dra. Salomé Alejandra Oriol-López

Av. Instituto Politécnico

Nacional Núm. 5260 ,

Col. Magdalena de las Salinas, Delegación Gustavo A.

Madero, México, D.F.

Tel: 57477560, ext. 738

E-mail: saorlopez@hotmail.com

Recibido para publicación: 13-05-2020

Aceptado para publicación:

26-06-2020
D esde su aparición en diciembre de 2019, la enfermedad por coronavirus SARS-CoV-2 o COVID-19 se ha diseminado rápidamente por el mundo con $8,108,641$ casos confirmados y 438,583 defunciones. En México estas cifras alcanzan 150,264 casos confirmados y 17,580 fallecimientos al 15 de junio de 2020. La infección por COVID-19 grave está asociada a coagulopatía; la historia clínica debe ser exhaustivamente revisada, los pacientes con comorbilidades pueden tener riesgo mayor de alteraciones de la coagulación. Las reacciones inflamatorias locales y sistémicas estimuladas por el virus afectan la función de las células del endotelio vascular induciendo daños en la pared, contribuyendo al estado de hipercoagulabilidad, inmovilización prolongada con la consiguiente estasis venosa, completa la tríada de Virchow. Reportes mencionan incrementos en la incidencia de embolia pulmonar, señalando que el virus SARS-COV-2 infecta y daña el tejido endotelial tanto in vivo como in vitro, pudiendo ser el origen de la coagulopatía, que activa la hemostasia guiando a trastornos trombóticos, estos pacientes suelen desarrollar trombocitopenia (36.2\%), elevación de dímero-D (46.4\%), en casos graves (57.7 y $59.6 \%)$. El endotelio tiene un papel primordial; primero, la hipoxia observada en COVID-19 induce a vasoconstricción, disminuye el flujo sanguíneo con la consecuente disfunción endotelial; segundo, la hipoxia puede desplazar el fenotipo antitrombótico y antiinflamatorio del endotelio basal hacia un fenotipo proinflamatorio y procoagulante; tercero, en relación con COVID-19 las citosinas proinflamatorias llevan a lesión endotelial, liberando el factor de Von Willebrand involucrado en la hemostasia primaria y en la sobreexpresión del factor tisular, que actúa como puente entre las plaquetas activadas ${ }^{(1-4)}$. 
Se evaluará con precisión la funcionalidad de los factores de la coagulación en todos los pacientes: a) el sistema exógeno con el tiempo de protrombina (TP) o la relación internacional estandarizada (INR): tiempos prolongados indican una cantidad o calidad anormal de los factores exógenos del sistema de coagulación o bien la presencia de anticoagulantes sanguíneos; b) el endógeno: cuando se prolonga el tiempo de tromboplastina parcial activada (TTPa), revela calidad o cantidad anormal, o presencia de sustancias anticoagulantes séricas. Si se acorta revela hipercoagulación; también lo podemos utilizar para establecer las dosis de heparina; c) la vía común de la coagulación mediante: el tiempo de trombina (TT) y fibrinógeno. El TT alargado y fibrinógeno normal señala la presencia de sustancias anticoagulantes sanguíneas; la prolongación de TT y fibrinógeno disminuido indican hipofibrinogenemia; d) el sistema fibrinolítico: dímeros-D

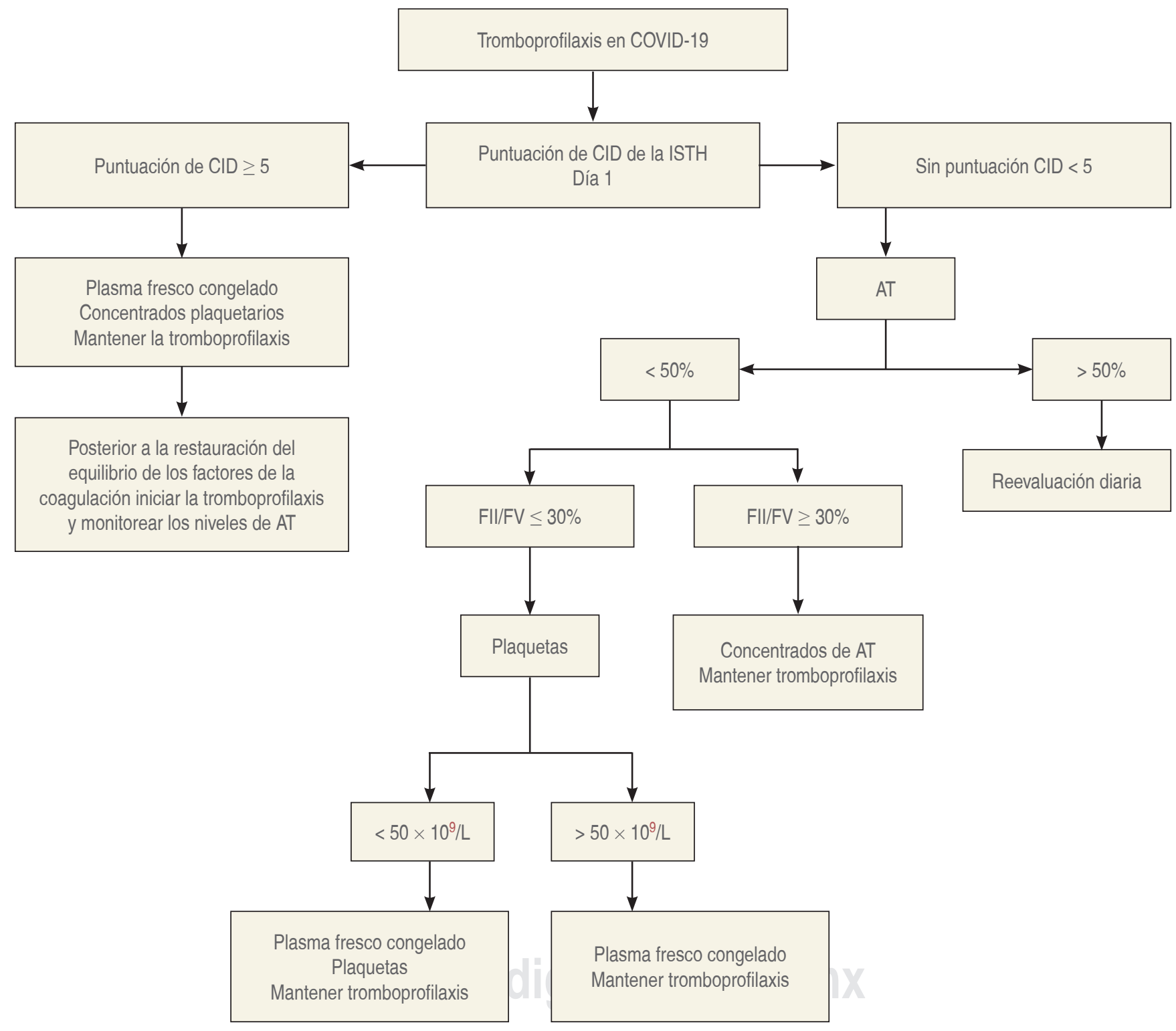

Figura 1: Algoritmo de tromboprofilaxis en pacientes con COVID-19 grave con alteraciones de la coagulación. Desde el ingreso del paciente evaluar alteraciones de la coagulación, de acuerdo a la evolución.

CID = coagulación intravascular diseminada; ITSH = Sociedad Internacional de Trombosis y la Hemostasia; AT = antitrombina; FII = Factor II; FV = Factor V. Valores 5 el paciente cursa con CID.

Adaptado de: Terpos E, Ntanasis-Stathopoulos I, Elalamy I, Kastritis E, Sergentanis TN, Marianna PM, et al. Hematological findings and complications of COVID-19. Am J Hematol. 2020;95:834-847. doi: 10.1002/ajh.25829. 


\begin{tabular}{lcc}
\multicolumn{2}{c}{ Tabla 1: Criterios para diagnosticar } \\
coagulación intravascular diseminada. \\
Puntuación & ITSH \\
\hline Plaquetas $\left(\times 10^{\circ}\right)$ & 0 & $>100$ \\
& 1 & $\leq 100$ \\
TP/TTPa (s) & 2 & $\leq 50$ \\
& 0 & TP prolongado $<3$ \\
Indicadores relacionados a fibrina (mg/L) & 1 & TP $\geq y \leq 6$ \\
& 2 & $\mathrm{TP} \geq 6$ \\
& 0 & $\mathrm{DD}<2.5$ \\
Fibrinógeno (g/L) & 2 & $\mathrm{DD} 2.5 \leq 5$ \\
& 2 & $\mathrm{DD}<5$ \\
Desórdenes subyacentes que se asocian & 0 & $\mathrm{DD} \geq 5.0$ \\
con CID & 0 & $\geq 1.0$ \\
Puntaje Total & 1 & $<1.0$ \\
\hline
\end{tabular}

ITSH = Sociedad Internacional de Trombosis y la Hemostasia; TP = tiempo de protrom bina; $\mathrm{TPPTa}=$ tiempo parcial de tromboplastina activado; $\mathrm{DD}=$ dímero $\mathrm{D}$. Valor total $\leq$ 5 seguir vigilando parámetros.

Adaptado de: Song JC, Wang G, Zhang W, Zhang Y, Wei-Qin Li W-C, Zhou Z. Chinese expert consensus on diagnosis and treatment of coagulation dysfunction in COVID19. Military Med Res. 2020;7. https://doi.org/10.1186/s40779-020-00247-7.

(DD) y productos de degradación de la fibrina (PDF) incrementados en estos parámetros sugieren actividad del sistema fibrinolítico; e) recuento plaquetario (PLT): si disminuye el número aumenta el riesgo de sangrado, si el número aumenta existe propensión a la adhesión, agregación y reacciones de liberación, incrementando el riesgo de trombosis.

Podemos encontrar: aumento de factores procoagulantes como el fibrinógeno, además de gran elevación del DD, niveles superiores a 1,000 ng/mL están relacionados con incremento en la mortalidad, también se prolonga el $\mathrm{TP}^{(5)}$ (Figura 1).

Al progresar la enfermedad puede presentarse linfopenia, aumento de ferritina, DD, lactato deshidrogenasa (LDH) así como anormalidades de la coagulación principalmente en TP (prolongado) y TPTa, las plaquetas y los neutrófilos tienden a disminuir, se observa un incremento de la proteína $C$ reactiva; la interleucina-6 (IL-6) transaminasas, los triglicéridos, NT- pro BNP, la troponina, la creatinina y la urea sérica tienden a disminuir ${ }^{(3,6)}$.

La coagulación intravascular diseminada (CID) tiene la característica de ser secundaria a daños locales, siendo la manifestación del fracaso de la coagulación y el enlace intermedio en el desarrollo de la falla orgánica múltiple (FOM). El diagnóstico se basa en la puntuación de la Sociedad Internacional de Trombosis y la Hemostasia (ISTH por sus siglas en inglés) que incluyen del TP, PLT, fibrinógeno y DD, una puntuación $\geq 5$ se clasifica como CID abierta y $<5$ como no abierta, la que se evaluará diariamente ${ }^{(7)}$ (Tabla 1).

El uso de TEG y Sonoclot consigue evaluar el estado general de la coagulación, mostrando buena correlación con la evaluación de los factores de coagulación fibrinógeno y PLT en pacientes críticos. El analizador automatizado de la coagulación demuestra mayor sensibilidad en el monitoreo de factores de la coagulación y función plaquetaria, mientras que TEG muestra grandes ventajas en el monitoreo de la función fibrinolítica ${ }^{(3,6-8)}$.

El cuadro grave presenta trombosis, lo más probable es que la enfermedad sea un estado hipercoagulable, los exámenes de coagulación de rutina pueden presentar TP acortado, TTPa, pruebas viscoelásticas con hiperfunción de factores de la coagulación y plaquetaria; el incremento significativo del DD señala la posibilidad de trombosis, por lo que la terapia anticoagulante se recomienda en pacientes sin contraindicaciones para la anticoagulación, prefiriendo los anticoagulantes parenterales con elección del fármaco y la dosis de acuerdo a la gravedad y ubicación de la embolia, se elige la heparina no fraccionada/ de bajo peso molecular (HBPM) para disminuir el agotamiento de los sustratos de la coagulación. En estos enfermos, la «tormenta de citocinas» activa la coagulación con el consecuente consumo excesivo de los factores de coagulación y plaquetas; el agotamiento excesivo de los sustratos provoca la disfunción que a su vez conduce al desarrollo de CID e incluso FOM, por lo que la sugerencia es administrar de una a dos dosis de HBPM diariamente hasta que el DD regrese a la normalidad. Si los PDF $\geq 10 \mathrm{~g} / \mathrm{mL}$ y/o DD $\geq 5.5 \mu \mathrm{g} / \mathrm{mL}$ no usar esta dosis, emplear heparina (3-15 UI/kg por hora), reevaluando a las cuatro horas los factores de la coagulación y plaquetas ${ }^{(9,10)}$.

Dentro de las ventajas de utilizar heparina no fraccionada se encuentran: vida media corta, proceso de monitoreo conve-

\begin{tabular}{|c|c|c|c|c|}
\hline Dosis de carga (UI/kg) & Dosis de mantenimiento (UI/kg*h) & INR & TTPa (s) & $\operatorname{PLT}\left(x 10^{9}\right)$ \\
\hline $30 \sim 50$ & 10 & $\leq 1.5$ & $\leq 40$ & $\geq 150$ \\
\hline $20 \sim 40$ & 5 & $>1.5 \leq 2.5$ & $>40 \leq 60$ & $<150 \geq 70$ \\
\hline No & $\begin{array}{c}\text { Anticoagulación con citrato o sin } \\
\text { anticoagulación }\end{array}$ & $>2.5$ & $>60$ & $<70$ \\
\hline
\end{tabular}

INR = relación internacional normalizada; TPTa = tiempo parcial de tromboplastina activado; $\mathrm{PLT}=$ plaquetas; $\mathrm{UI}=$ unidades internacionales ${ }^{(5)}$. 
niente, además se neutraliza con protamina, por lo que debería ser la primera elección, hay que administrarla vía endovenosa, determinando la dosis de acuerdo al órgano afectado y a la función de la coagulación, en disfunción grave iniciar con dosis baja $1 \mathrm{UI} /\left(\mathrm{kg}^{*} \mathrm{~h}\right)$ para titular adecuadamente evaluando resultados de laboratorio. La HBPM no es fácil de ajustar o monitorear por su vida media larga, por lo que se establece su uso en cuadros de coagulopatía de leve a moderado; la dosis inicial a suministrar es $1 \mathrm{mg} / \mathrm{kg}$ cada 12 horas vía endovenosa o subcutánea, monitoreando la dosis de acuerdo con la actividad anti-Xa con un rango de 0.6 1.0 UI/mL. Al metabolizarse en los riñones, monitorearla en insuficiencia renal. La actividad anti-trombina III debe mantenerse $>80 \%$, de lo contrario puede afectar el efecto anticoagulante, si disminuye la actividad se puede aumentar empleando PFC. Además, la heparina tiene un efecto antiinflamatorio, protege las células endoteliales, reduce la toxicidad de las histonas en la unión endotelial, disminuye el edema pulmonar y la fuga vascular ${ }^{(11,12)}$ (Tabla 2).

La hemorragia no es común en estos pacientes, cuando ocurre en la CID asociada a esta enfermedad, el soporte con hemoderivados debe considerarse como en coagulopatía séptica: concentrado plaquetario para mantener el recuento de plaquetas mayor de 50,000/L en CID, con sangrado activo mayor de 20,000; en personas con alto riesgo de sangrado, o que requieren de procedimientos invasivos plasma fresco congelado (PFC) a dosis de $15-25 \mathrm{~mL} / \mathrm{kg}$; en pacientes con hemorragia activa con relaciones prolongadas de TP y/o ТPTa (> 1.5 veces lo normal) o disminución de fibrinógeno $(<1.5 \mathrm{~g} / \mathrm{L})$; concentrado de fibrinógeno o crioprecipitado en pacientes con hipofibrinogenemia severa persistente $(<1.5$ $\mathrm{g} / \mathrm{L}$ ) y concentrado de complejo de protrombina (CCP) si la transfusión de PFC no es posible, de acuerdo con la evidencia disponible el ácido tranexámico no debe emplearse de rutina en la CID asociada a COVID-19(5,13).

La prueba comparativa de heparinasa TEG se utiliza para evaluar la función de la coagulación en pacientes sometidos a terapia anticoagulante con heparina, además sirve para controlar la dosis de heparina no fraccionada. La prueba requiere del tubo control y del tubo de heparinasa que se probarán al mismo tiempo; el control representa los efectos combinados de la disfunción de la coagulación propia del paciente y el efecto de la heparina, la prueba de heparinasa puede reflejar con exactitud el estado de coagulación del propio paciente al descomponer la heparina en sangre, por tanto, puede evaluar la dosis de heparina a usar. La proporción de tiempo $\mathrm{R}$ en el tubo de control al tiempo $\mathrm{R}$ en el tubo de heparinasa es de aproximadamente $2^{(5)}$.

En la terapia sustitutiva renal continua (TRSC) los métodos de anticoagulación se dividen en sistémica, local y sin anticoagulación; la sistémica disminuye la coagulación mediante el uso de fármacos anticoagulantes endovenosos; la local reduce la coagulación en el circuito extracorpóreo sin afectar la coagulación sanguínea, dependiendo del objetivo se utilizará sistémica o local, ajustando la dosis según el estado de coagulación del paciente, evitando riesgo de sangrado mayor, monitoreando el estado dentro de las seis horas de administración del anticoagulante (heparina no fraccionada) después realizar el monitoreo dependiendo de la severidad de su condición. Efectuar prueba de comparación de heparinasa con la finalidad de evaluar la dosis de heparina, considerar el uso de protamina en caso de sobredosis. Repetir la HBPM en bolo o en infusión, el bolo inicial debería ser de 60-80 UI/kg, 20-30 min antes del tratamiento, con dosis adicional de 30 40 UI cada cuatro a seis horas, disminuyendo gradualmente las dosis a medida que el período de tratamiento continúe, midiendo la actividad plasmática anti-Xa y manteniéndola a $0.3 \mathrm{UI} / \mathrm{mL}$. Si se presenta sangrado activo la TSRC, realizar anticoagulación tópica con citrato para evitar trastornos agravantes de la coagulación. La concentración de calcio libre después del filtro debe mantenerse a $0.25-0.35 \mathrm{mmol} / \mathrm{L}$, el calcio libre venoso debe estar a 1.1-1.35 mmol/L, la dosis de solución de cloruro o gluconato de calcio debe ajustarse dependiendo de la concentración sérica de calcio ionizado ${ }^{(14)}$.

En pacientes críticamente enfermos con COVID-19 en quienes el daño pulmonar es importante, el tratamiento indicado es la oxigenación mediante membrana extracorpórea (ECMO), la heparina es el anticoagulante comúnmente usado, aumenta el riesgo de sangrado, éste puede ser severo en el momento de realizar la intubación para ECMO o en la incisión quirúrgica nasal, en vías respiratorias, sangrado gastrointestinal y el más grave el intracraneal; el monitoreo de la coagulación incluye: el tiempo de coagulación activado (ACT) y TTPa; mantener ACT entre 180-220 s y/o ACT y TTPa mantenerlos a 1.5 veces el límite superior, también se puede monitorear con la prueba de comparación de heparinasa TEG. Si empleamos la actividad anti-Xa para controlar la dosis de heparina mantenerla en $0.3-0.7 \mathrm{UI} / \mathrm{mL}^{(5,14)}$.

La terapia de reemplazo dirigida por objetivos implica emplear indicadores de la coagulación de rutina, parámetros de TEG e índices de Sonoclot. Cuando el TP o TTPa se prolonga $>1.5$ veces o el tiempo $\mathrm{R}$ del TEG es $>10$ min o el Sonoclot es $>240$ s (sangre anticoagulada), se debe infundir PFC de 15 a $30 \mathrm{~mL} / \mathrm{kg}$ en cuanto sea posible, monitoreando los indicadores de la coagulación para determinar dosis adicionales, si existe sobrecarga de líquidos, los factores de coagulación pueden complementarse en combinación con concentrado de complejo de protrombina. Si el fibrinógeno $<1.5 \mathrm{~g} / \mathrm{L}$ o el TEG fibrinógeno es funcional, la prueba del indicador de nivel FFma es $<10 \mathrm{~mm}$ o el CR del Sonoclot $<10$, podemos infundir crioprecipitado de fibrinógeno a dosis de $10 \mathrm{~mL} / \mathrm{kg}$ o fibrinógeno humano $30-50$ $\mathrm{mg} / \mathrm{kg}$, monitoreando los indicadores de la coagulación para mantener el fibrinógeno plasmático en $1.5 \mathrm{~g} / \mathrm{L}$ al menos ${ }^{(5,8)}$.

En pacientes sin sangrado se sugiere la transfusión de plaquetas si la cuenta es $<20 \times 10^{9} / \mathrm{L}$; en pacientes que podrían 
someterse a una punción lumbar o presenten sangrado activo, se recomienda la transfusión de concentrados plaquetarios si el recuento es $<50 \times 10^{9} / \mathrm{L}$, si el paciente está bajo ECMO y el recuento plaquetario es $<80 \times 10^{9} / \mathrm{L}$ habrá que transfundir concentrados plaquetarios. El empleo de concentrados plaquetarios aún es controvertido; no obstante, en pacientes que están en terapia antiplaquetaria, se recomienda la transfusión en casos de sangrado persistente con disfunción plaquetaria e incluso trombocitopenia. Individualizar la dosis, la que generalmente es una unidad de aféresis de plaquetas o dosis equivalente de concentrado plaquetario por dosificación; transfundir $\geq 2$ unidades de aféresis si el sangrado es amenaza grave para la vida, el objetivo principal es transfundir la dosis mínima requerida para mantener el recuento plaquetario. Teóricamente una unidad de plaquetas puede aumentar desde $4 \times$ $10^{9} / \mathrm{L}$ hasta $8 \times 10^{9} / \mathrm{L}$ en un adulto de $70 \mathrm{~kg}^{(5,15,16)}$.

Pacientes con COVID-19 grave con encefalopatía hepática grado II o superior con las siguientes manifestaciones pueden diagnosticarse con insuficiencia hepática aguda: a) anorexia, distensión abdominal, náuseas o vómitos; b) ictericia progresiva con bilirrubina sérica total $\geq 10$ veces el límite superior al normal en poco tiempo o un aumento diario $\geq 17 \mu \mathrm{mol} / \mathrm{L} ; \mathrm{c}$ ) actividad de protrombina $\leq 40 \%$ o INR 1.5 excluyendo otras causas; d) contracción hepática progresiva.

En COVID-19 grave con coagulopatía que experimenta insuficiencia hepática, se recomienda el uso del sistema de soporte artificial hepático (ALSS, por sus siglas en inglés); utilizan el intercambio de plasma: desechando el plasma que se separó de las células sanguíneas por centrifugación, luego se reemplaza por plasma recién congelado, eliminando sustancias de gran peso molecular como los autoanticuerpos, complejos inmunes, colesterol, bilirrubinas, fármacos y venenos, complementando los factores de coagulación ${ }^{(5,14)}$.

En los catéteres venosos centrales utilizados para controlar el estado hemodinámico administrar nutrición parenteral, medicamentos, hemoderivados y realizar hemodiálisis; los reportes indican que existe oclusión de éstos e incluso en TRSC y ECMO se obliteran los circuitos asociados al anticuerpo lúpico. En ocasiones un catéter intrarterial también se coloca para un mejor control de la presión arterial cuando existe inestabilidad hemodinámica; para lograr la funcionalidad de los dispositivos de acceso vascular las pautas actuales recomiendan enjuagar con heparina; sin embargo, ésta aumenta los tiempos de coagulación cuando las dosis son demasiado altas provocando hemorragias fatales. El enjuagado frecuente también resulta en alteración de las pruebas de coagulación y perturba el monitoreo de la dosis de heparina, por lo que se exhorta evitar enjuagar con heparina los accesos vasculares en casos graves de COVID-19 y coagulopatía ${ }^{(17,18)}$.

Estudios previos demostraron que la infusión de $1 \mathrm{~L}$ de solución salina normal puede aumentar en promedio 6\% el
RC en Sonoclot y disminuir 15\% de TCA; con la succinil gelatina puede reducir el RC en $12 \%$ y alargar el tiempo pico en 96\%, tendiendo a la hipocoagulación. A medida que se incrementa el volumen de infusión del líquido, la disfunción en la coagulación se agrava aún más debido al efecto de dilución, la dilución isovolumétrica resulta en la disminución de la agregación plaquetaria, por lo tanto, en COVID-19 grave y coagulopatía el control estricto de infusión de líquidos, especialmente de coloides sintéticos es primordial ${ }^{(5)}$.

Aún no hay reportes en la literatura de procedimientos quirúrgicos de urgencia en pacientes COVID-19 grave con coagulopatía, existen de operación cesárea; los lineamientos para pacientes sin COVID-19 serán similares a los que requieren cirugía o procedimiento invasivo de emergencia, lo importante es determinar el estado de coagulación del paciente como el INR cuando hay terapia de antagonista de la vitamina K (AVK), TTPa para el dabigatrán, TP para rivaroxabán y probablemente edoxabán. Con valores de INR $<1.5$ puede ser intervenido, si es mayor habrá que evaluar la situación específica, si puede diferirse el procedimiento por ocho a 12 horas, administrar $10 \mathrm{mg}$ de vitamina K endovenosa, ante la emergencia suministrar complejo de concentrado de protrombina a 25-50 UI/kg dependiendo del INR actual y del objetivo. En el caso de anticoagulantes orales, si las pruebas muestran actividad anticoagulante (apixabán o edoxabán), se evaluará el estado clínico del paciente para determinar cuál es el tiempo máximo que puede diferirse la cirugía (idealmente 24 horas), en otros casos la opción es utilizar antagonistas, igualmente tomando en cuenta el procedimiento quirúrgico, hora de la última dosis así como la función renal. La anestesia neuroaxial está contraindicada, la anestesia general es la elección ${ }^{(19-21)}$ (Figura 2).

En los antagonistas en cirugía de emergencia o con sangrado activo el agente hemostático es el idarucizumab, que inhibe selectivamente la actividad anticoagulante del dabigatrán en minutos, se administran dos dosis de 2.5 g en infusión rápida con 15 min de diferencia, indicado en sangrado de órgano vital, agudo mayor y/o quien requiera cirugía o procedimiento invasivo de emergencia con alto riesgo de sangrado en las próximas ocho horas.

Las interacciones entre el tratamiento anticoagulante y los antivirales son por medio del citrocromo P450 (CYP) y la glicoproteína $\mathrm{P}$ (P-gp) principalmente. La heparina no fraccionada, la de bajo peso molecular (HBPM) y el fondaparinux pueden administrarse de manera segura con fármacos experimentales en COVID-19 porque no hay interacciones probadas ni esperadas, por otro lado, las probables propiedades de la heparina y sus derivados podrían ser útiles en esta situación. Los agentes dicumarólicos tienen interacciones con los inhibidores de la proteasa: atazanavir, lopinavir/ ritonavir y ribavirina. Concretamente los niveles séricos aumentan mediante la inhibición del CYP2C9 al coadministrar 


\section{Evaluación del estado de coagulación (pruebas cualitativas y cuantitativas específicas) \\ Dabigatrán: TTd $\pm T T P a$ \\ Rivaroxabán; anti-Xa $\pm \mathrm{TP}$ VKA: INR \\ Registrar la hora de la última dosis Cuenta sanguínea completa y función renal}

\begin{tabular}{|c|}
\hline Posponer la cirugía \\
Seguir los pasos para cirugía electiva \\
\hline \\
Anestesia neuroaxial: contraindicada \\
Medidas generales: oxigenoterapia, fluidoterapia, \\
mantener diuresis adecuada \\
Transfusión de hemoderivados: \\
Mantener Hb adecuada \\
Plaquetas: $75 \times 10^{9} / \mathrm{L}$ (SNC: $\left.100 \times 10^{9} / \mathrm{L}\right)$ \\
Agentes antagonistas: \\
Dabigatrán: idarucizumab (2.5 +2.5 g IV) \\
VKA: vitamina K (10 mg IV) \\
Anti-Xa: andexanet (bolo IV $400-800$ mg en 15 a 30 min \\
Infusión $480-960$ mg en 2 horas) \\
Agentes procoagulantes: \\
PCC: $25-50$ Ul/kg \\
OFC (VKA), FEIBA, factor VIla, etcétera
\end{tabular}

Para OADs, considerar diferir la cirugía por 1-2 vidas medias de eliminación del fármaco (12-24 horas) Para VKAs, esperar $48 \mathrm{~h}$ o administrar vitamina $\mathrm{K}$

Cirugía de urgencia o hemorragia activa

Figura 2: Propuestas de tratamiento en pacientes en terapia anticoagulante, quienes podrían necesitar procedimiento quirúrgico o invasivo de emergencia. TTd = tiempo de trombina diluido; TPTa = tiempo parcial de tromboplastina activado; SNC = sistema nervioso central; IV = intravenoso; AOD = anticoagulación oral directa; factor VIla: factor VII activado; PFC = plasma fresco congelado; INR = relación internacional normalizada; CCP = complejo concentrado de protrombina; TP = tiempo de protrombina; $\mathrm{VKA}=$ antagonista de vitamina $\mathrm{K} ; \mathrm{Xa}$ : factor $\mathrm{X}$ activado. ${ }^{*}$ Órgano crítico como SNC, pericardio, intraocular, intraarticular, síndrome compartamental muscular. Adaptado de: Vivas D, Roldán I, Ferrandis R, Marín F, Roldán V, Tello-Montoliu A, et al. Perioperative and Periprocedural Management of Antithrombotic Therapy: Consensus Document of SEC, SEDAR, SEACV, SECTCV, AEC, SECPRE, SEPD, SEGO, SEHH, SETH, SEMERGEN, SEMFYC, SEMG, SEMICYUC, SEMI, SEMES, SEPAR, SENEC, SEO, SEPA, SERVEI, SECOT and AEU. Rev Esp Cardiol. 2018;71:553-564.

atazanavir; con lopinavir/ritonavir y ribavirina disminuyen por estimulación de CYP2C9. Esperaríamos una probable interacción en la coadministración con tocilizumab, ya que puede interferir con CYP a través de la vía de la IL-6, es obligatorio un monitoreo estricto del INR.

Por su perfil de seguridad y mejor efectividad en comparación con los dicumarólicos, los anticoagulantes orales no antagonistas de la vitamina K (NOAC) son la opción válida, pero no están exentos del riesgo de interacciones sobre todo en pacientes con COVID-19, se contraindica la administración de inhibidores directos de Xa: apixabán, rivaroxabán y edoxabán conjunta con agentes antivirales, ya que inhiben el CYP3A4 (atazavir y lopinavir) y la P-gp eleva sus niveles séricos al doble con ritonavir. Al administrarse simultáneamente con cloroquina o hidroxicloroquina, estos fármacos se metabolizan por la misma vía de enzimas CYP, reduciendo la eliminación de estos anticoagulantes con el consecuente incremento en el riesgo de sangrado. El TCZ disminuye la inhibición mediada por IL-6 del CYP, elevando la exposición de inhibidores directos de Xa. El uso de dabigatrán, que es un inhibidor directo de la trombina, no se recomienda con atazanavir, al inhibir al CYP3A4 eleva sus concentraciones 
sanguíneas; en contraparte el suministro conjunto de lopinavir/ritonavir puede disminuir la exposición de dabigarán mediante la inhibición de la P-pg.

Aun cuando el metabolito activo para clopidogrel está formado principalmente por CYP2C19, la inhibición de CYP3A4 puede llevar a la disminución en la dosis efectiva de clopidogrel o aumentar los efectos de ticagrelor ${ }^{(22-24)}$.
En resumen, es importante conocer la evolución del paciente, tanto clínica como de los marcadores de la coagulación, para determinar la terapia a emplear en el momento en que sea solicitada para procedimiento invasivo o quirúrgico de emergencia, tomando en cuenta todas las precauciones mencionadas en el manejo perioperatorio recomendadas para evitar la contaminación del personal de la salud.

\section{REFERENCIAS}

1. Joly BS, Siguret V, Veyradier A. Understanding pathophysiology of hemostasis disorders in critically ill patients with COVID 19. Intensive Care Medicine. 2020. https://doi.org/10.1007/s00134-020-06088-1.

2. Debuc B, Smadja DM. Is COVID-19 a new hematologic disease? Stem Cell Rev and Rep. 2020. https://doi.org/10.1007/s12015-020-09987-4.

3. Fan BE, Chia YW, Lin SC, Kuperan P, Wei CS, Ling LM, et al. Global haemostatic tests in rapid diagnosis and management of COVID 19 associated coagulopathy in acute limb ischaemia. J Thromb Thrombolysis. 2020:1-6. https://doi.org/10.1007/s11239-020-02165-w.

4. Joly BS, Siguret V, Veyradier A. Understanding pathophysiology of hemostasis disorders in critically ill patients with COVID 19. Intensive Care Medicine. 2020. https://doi.org/10.1007/s00134-020-06088-1.

5. Song JC, Wang G, Zhang W, Zhang Y, Wei-Qin Li W-C, Zhou Z. Chinese expert consensus on diagnosis and treatment of coagulation dysfunction in COVID19. Military Med Res. 2020;7. https://doi.org/10.1186/s40779020-00247-7.

6. Wujtewicz MA, Dylczyk-Sommer A, Aszkiełowicz A, Zdanowski S, Piwowarczyk S, Owczuk R. COVID-19. ¿What should anaethesiologists and intensivists know about it? Anaesthesiol Intensive Ther. 220;52:3441. https://doi.org/10.5114/ait.2020.93756.

7. Thachil J, Tang N, Gando S, Falanga A, Cattaneo M, Levi M, Clark C, Iba T. ISTH interim guidance on recognition and management of coagulopathy in COVID-19. Journal of Thrombosis and Haemostasis 2020. doi: 10.1111/jth.14810.

8. Panigada M, Bottino N, Tagliabue P, Grasselli G, Novembrino C, Chantarangkul V, et al. Hypercoagulability of COVID-19 patients in Intensive Care Unit. a report of thromboelastography findings and other parameters of hemostasis. J Thromb Haemost. 2020. doi doi: 10.1111/ JTH.14850.

9. Terpos E, Ntanasis-Stathopoulos I, Elalamy I, Kastritis E, Sergentanis TN, Marianna PM, et al. Hematological findings and complications of COVID-19. Am J Hematol. 2020;95:834-847. doi: 10.1002/ajh.25829.

10. Tang N, Bai H, Chen X, Gong J, Li D, Sun Z. Anticoagulant treatment is associated with decreased mortality in severe coronavirus disease 2019 patients with coagulopathy. J Thromb Haemost. 202018:1094-1099. doi: 10.1111/JTH.14817.

11. Levi M, Scully M. How I treat disseminated intravascular coagulation. Blood. 2018;131:845-854.

12. Thachil J. The versatile heparin in COVID-19. J Thromb Haemost. 2020;18:1020-1022. doi: 10.1111/jth.14821.
13. Connors JM, Levy JH. COVID-19 and its implications for thrombosis and anticoagulation. Blood. 2020;135:2033-2040.

14. Bikdeli B, Madhavan MV, Jiménez D, Chuich T, Dreyfus I, Driggin E, et al. COVID-19 and thrombotic or thromboembolic disease: implications for prevention, antithrombotic therapy, and followup: JACC state-of-the-art review. Journal of the American College of Cardiology. 2020;75:2950-2973. https://doi.org/10.1016/j. jacc.2020.04.031.

15. Baron DM, Franchini M, Goobie SM, Javidroozi M, Klein AA, Lasocki S, et al. Patient blood management during the COVID-19 pandemic: a narrative review. Anesthesia 2020. doi: 10.1111/anae.15095.

16. Galluccio F, Ergonenc T, Garcia MA, El-Sayed AA, Pérez-Herrero M, Aguilar R, et al. Treatment algorithm for COVID-19: a multidisciplinary point of view. https://doi.org/10.1007/s10067-020-05179-0.

17. Thachil J, Agarwal S. Understanding the COVID-19 coagulopathy spectrum. doi: 10.1111/anae.15141.

18. Harzallah I, Debliquis A, Drénou B. Frequency of lupus anticoagulant in Covid-19 patients. J Thromb Haemost. 2020. doi: 10.1111/JTH.14937.

19. Vlachodimitropoulou KE, Vivanti AJ, Shehata N, Benachi A, Le Gouez A, Desconclois C, et al. COVID-19 and acute coagulopathy in pregnancy. doi: 10.1111/jth.14856.

20. Vivas D, Roldán I, Ferrandis R, Marín F, Roldán V, Tello-Montoliu A, et al. Perioperative and Periprocedural Management of Antithrombotic Therapy: Consensus Document of SEC, SEDAR, SEACV, SECTCV, AEC, SECPRE, SEPD, SEGO, SEHH, SETH, SEMERGEN, SEMFYC, SEMG, SEMICYUC, SEMI, SEMES, SEPAR, SENEC, SEO, SEPA, SERVEI, SECOT and AEU. Rev Esp Cardiol. 2018;71:553-564.

21. Oprea AD, Noto CJ, Halaszynski TM. Risk stratification, perioperative and periprocedural management of the patient receiving anticoagulant therapy. J Clin Anesth. 2016;34:586-599.

22. Canonico ME, Siciliano R, Scudiero F, Sanna GD, ParodiM G. The tugof-war between coagulopathy and anticoagulant agents in patients with COVID-19. Eur Heart J Cardiovasc Pharmacother. 2020. doi: 10.1093/ ehjcvp/pvaa048.

23. Tang N, Li D, Wang X, Sun Z. Abnormal coagulation parameters are associated with por prognosis in patients with novel coronavirus pneumonia. J Thromb Haemost. 2020;18:844-847.

24. Cecconi M, Forni G, Mantovani A. Ten things we learned about COVID 19. Intensive Care Med. 2020;1-4. https://doi.org/10.1007/s00134-02006140-0. 